\title{
A Case Report of Haemolysis in a G6PD Deficient Child Due to Acetaminophen Overdose Resulted from Parental Unawareness
}

\author{
George Mathew Panachiyil', Babu Tirin', Sebastian Juny ${ }^{1,{ }^{*},}$, Mandyam Ravi Dhati ${ }^{2}$ \\ 'Department of Pharmacy Practice, JSS College of Pharmacy, JSS Academy of Higher Education and Research, Mysuru, Karnataka, INDIA. \\ 2Department of Paediatrics, JSS Medical College and Hospital, JSS Academy of Higher Education and Research, Mysuru, Karnataka, INDIA.
}

\begin{abstract}
Although acetaminophen is considered a low-risk analgesic and antipyretic drug in Glucose-6-Phosphate Dehydrogenase (G6PD) Deficiency patients, cases of haemolysis following acetaminophen overdose have been published in the literature. An eleven-month-old boy was admitted to the paediatrics department of the hospital with the complaints of haematuria and yellowish discolouration of the eyes since one day. He had been previously diagnosed as G6PD deficiency. Unknowingly the mother of the child simultaneously given three different brands of acetaminophen prescribed by different paediatricians at various times resulted in an overdose of acetaminophen. On admission, the child had haemoglobin of $4 \mathrm{~g} / \mathrm{dl}$. The laboratory results were suggestive of haemolytic process. Direct Coombs test was negative. Acetaminophen overdose was the most likely cause of the haemolysis. The child was managed conservatively and no further episodes of haematuria was observed. Naranjo and WHO causality assessments were done, indicating a probable relationship between the patient's symptoms and the use of acetaminophen. Acetaminophen overdose induced haemolysis due to G6PD deficiency is an unusual complication which is generally
\end{abstract}

reported in children. The majority of G6PD deficient individuals remains asymptomatic until a trigger (during certain infections or fava beans or certain drugs) induces haemolysis. We document a case report of acetaminophen overdose induced haemolysis in a baby with G6PD deficiency and conclude that there is a need for parental education about the drugs that can trigger haemolysis in G6PD deficient children and also about the different brands of the same drug available in the market.

Key words: Acetaminophen, Paracetamol, NSAIDs, G6PD deficiency, Haemolysis, Anaemia.

\section{Correspondence}

Mrs. Juny Sebastian, Lecturer in Department of Pharmacy Practice, JSS College of Pharmacy, JSS Academy of Higher Education and Research, Mysuru, Karnataka - 570015, INDIA.

Phone: +91 9620976279

Email: junysebastian@jssuni.edu.in

DOI: 10.5530/jyp.2019.11.46

\section{INTRODUCTION}

Glucose-6-Phosphate Dehydrogenase (G6PD) deficiency is an X-linked, inherited human enzyme defect carrying a mutation in the G6PD gene that causes deficiency of the enzyme. ${ }^{1,2}$ G6PD deficient individuals are at risk of haemolytic anaemia, which usually occurs when Red Blood Cells (RBCs) undergo oxidative stress triggered by ingestion of certain drugs or fava beans or during certain infections. ${ }^{3}$ Although many factors are involved, drug induced haemolysis is one of the most common adverse clinical consequence of G6PD deficiency. ${ }^{2}$ More than thirty drugs have been linked to acute haemolysis in G6PD deficient individuals. ${ }^{1}$ Acetaminophen, a Non-Steroidal Anti-Inflammatory Drug (NSAID) is a safe antipyretic-analgesic in normal therapeutic range in patients with G6PD deficiency. ${ }^{2}$ About $90 \%$ of ingested acetaminophen is metabolised via glucuronide or sulphide conjugation in the liver for urinary excretion. ${ }^{4}$ The remaining $10 \%$ is metabolised via the cytochrome $\mathrm{P} 450$ system to $\mathrm{N}$-acetyl-P-Benzoquinone Imine (NAPQI) and that is a potent oxidizing agent. ${ }^{4}$ In therapeutic range of acetaminophen, NAPQI is reduced via conjugation with glutathione to a nontoxic substance and is excreted. ${ }^{4}$ In the setting of acetaminophen overdose, haemolysis of G6PD-deficient red cells occurs from an increased susceptibility to oxidative damage as reduction of Nicotinamide Adenine Dinucleotide Phosphate (NADP+) to Nicotinamide Adenine Dinucleotide Phosphate Hydrogen (NADPH) is not happening at the normal rate. ${ }^{3,5} \mathrm{NADPH}$ is necessary to maintain the reduced form of glutathione. ${ }^{5}$ During this reaction reduced glutathione is oxidised rapidly, glutathione pools are exhausted, haemoglobin is denatured and heinz bodies are formed. The formed heinz bodies de- stroy membranes of RBCs that leads to haemolysis. ${ }^{3}$ The most effective management strategy of G6PD deficiency is to prevent haemolysis by avoiding oxidative stressors. ${ }^{1}$ There are several case reports of haemolysis as a potential complication after acetaminophen overdose in G6PD deficient individuals. ${ }^{6-10}$ We report a case of haemolysis attributed to acetaminophen overdose in a patient with known G6PD deficiency.

\section{CASE REPORT}

An eleven-month-old boy, known case of G6PD deficiency diagnosed at one-month age, was brought to the hospital with an acute haemolytic episode. For the past four days, the child had a history of cough, hurried breathing and fever. The parent had shown the child to three doctors in last three days and was prescribed an acetaminophen syrup at each visit. As the trade names of acetaminophen syrup prescribed at each visit were different, the parent did not know it was the same drug prescribed at all consultations and the child was administered with all three different brands of acetaminophen together for three days. In addition, the child also received a fixed drug combination of decongestant (Chlorpheniramine maleate $1 \mathrm{mg}+$ Phenylephrine 5mg + Acetaminophen $125 \mathrm{mg}+$ Sodium Citrate $60 \mathrm{mg}$ ). The child received $1.35 \mathrm{~g}$ per day of acetaminophen for three consecutive days. After three days of the above symptoms, the child also developed yellowish discolouration of eyes, haematuria and pallor. Though the child received an overdose of acetaminophen, the dose did not reach the toxicity level of $1.5 \mathrm{~g}(150 \mathrm{mg} / \mathrm{kg} /$ day $)$. Other than haemolysis the child did not have any of other complications. 
Vital signs on admission revealed a temperature of 37.78 degrees celsius, heart rate of 138 beats per minute, respiratory rate of 58 breaths per minute, blood pressure of $90 / 60 \mathrm{mmHg}$, capillary filling time less than $3 \mathrm{sec}$ and $\mathrm{SpO}_{2}$ was $85 \%$ at room air. His physical examination revealed for lethargy, pallor and icterus. Systemic examination revealed liver two centimetres below right costal margin and respiratory examination revealed rhonchi and crepts all over lung fields. Chest x-ray showed features suggestive of bronchiolitis. Haemoglobin was $4 \mathrm{~g} / \mathrm{dl}$. Peripheral blood smear was suggestive of haemolytic process. Anti-Hepatitis C virus, Hepatitis B surface antigen and rapid test for human immunodeficiency virus were negative. The child was initially transfused with one pint $(120 \mathrm{ml})$ packed RBCs. He was managed conservatively: maintenance IV fluids with bicarbonate for alkalinisation of urine, levosalbutamol and ipratropium nebulisations, ORS 1:200 (sos) were also given.

On hospital day two, haemoglobin was $7 \mathrm{~g} / \mathrm{dl}$, total bilirubin $3.98 \mathrm{mg} / \mathrm{dl}$, C-reactive protein $6.9 \mathrm{mg} / \mathrm{dl}$, Ig $2.5 \%$, NRBC $3.1 \%$, PCT $0.59 \%$, PDW 7.8fl, RDW 19.1\%, RDW-CV 19.1\%, RDW-SD 46.1fl, MCH 19.9pg, MCHC $28.4 \mathrm{~g} / \mathrm{dl}$, MCV 70.1fl, Reticulocyte count 5.2\%, Platelet count 6.74lakh/cumm, RBC count 2.11 million /cumm, PCV $14.8 \%$ and ESR $40 \mathrm{~mm}$ in $1 \mathrm{~h}$. Urine analysis showed presence of urine albumin $1+$. Peripheral blood smear impression was made as microcytic hypochromic anaemia with neutrophilic leucocytosis and thrombocytosis in haemolytic process. Liver function tests and serum electrolytes were in normal range. Direct Coombs test was negative. The child was transfused with one more pint $(120 \mathrm{ml})$. The final diagnosis made was G6PD deficiency with bronchiolitis with acute haemolytic episode precipitated by acetaminophen overdose.

On hospital day four, haemoglobin was $11.1 \mathrm{~g} / \mathrm{dl}$, Platelet count was 4.17 lakh/cumm and packed cell volume was $33.3 \%$. During the course of the hospital stay, the child improved symptomatically and no further episodes of haematuria was observed. The patient was discharged and counselling was provided to the parent.

\section{DISCUSSION}

Inherited and acquired factors affect individual susceptibility to and severity of, drug- induced oxidative haemolysis in G6PD deficiency. The three main triggers for haemolysis in G6PD deficient individuals are certain infections, fava beans and certain drugs. It is often difficult to establish a specific drug directly causes haemolytic crisis in G6PD deficient patients. First, as pharmacokinetics of drugs can vary between individuals, so a drug considered to be safe for some G6PD deficient individuals is not necessarily safe for all patients. Second, patients with an underlying clinical condition such as infection are sometimes treated using drugs which have potential oxidant effects that could lead to haemolysis. Third, patients are often using more than one type of medication. Fourth, Haemolysis in G6PD deficiency is a self-limiting process and therefore does not always produce clinically significant symptoms of anaemia and reticulocytosis. ${ }^{1}$ Among NSAIDs, the exact mechanism behind acetaminophen overdose induced haemolysis in G6PD deficiency has not been fully understood. G6PD and NADPH are crucial factors for protection of RBCs from oxidative damage and peroxides. NADPH is necessary to maintain reduced glutathione (GSH) and oxidized glutathione (GSSH) in the ratio 500:1. Reduced glutathione plays an important role in detoxification by reacting with hydrogen peroxides and organic peroxidases as well as in maintaining the cysteine residues of haemoglobin and other cell proteins in the reduced state. Reduced glutathione and catalase enzymes are necessary for the removal of peroxides which is mediated through the activation of NADPH. There is a fall in levels of GSH and NADPH observed in G6PD deficient individuals. In the presence of acetaminophen overdose, there is an aggravation in the increased susceptibility of RBCs to oxidative damage in G6PD deficient individuals and worsening of haemolysis. ${ }^{3,5,11}$ Wright et al. reported a case of haemolytic anaemia after acetaminophen overdose in a patient with G6PD deficiency. ${ }^{6}$ There were also two case reports of acetaminophen overdose followed by haemolysis, the difference from our case report is that it was not known that the patient was G6PD deficient at the time of hospital admission. ${ }^{8,9}$ All of these patients experienced jaundice 1-2 days after ingestion of acetaminophen, along with unconjugated hyperbilirubinaemia and decreased haemoglobin and haematocrit. Sklar et al. also reported a case of haemolysis as a potential complication of acetaminophen overdose in a patient with G6PD deficiency and in his report jaundice was not present for the patient. ${ }^{7}$ In S.S. Rickner et al. case report, the patient presented jaundice at the time of admission and also the patient developed hepatotoxicity thirty-three hour post ingestion in addition to a haemoglobin decline of $5 \mathrm{~g} / \mathrm{dl}$ in four days despite resolving hepatotoxicity. ${ }^{10}$ Our case report had a similar finding to the previous case reports. A number of factors favours acetaminophen as the probable cause of haemolysis such as evidence from previous case reports and the other drugs (Chlorpheniramine maleate, Phenylephrine, Sodium Citrate) that the patient taken have not been reported to cause haemolysis in G6PD deficient patients.

The causality assessment was done using both WHO causality assessment algorithm and Naranjo scale. Based on a time temporal relationship and the reaction unlikely attributed to other concurrent disease or medication, it was probable according to the WHO scale and same with Naranjo scale with a score of seven. The reaction was not predictable as frequency is not defined for the same. It is definitely preventable as this is a case of acetaminophen overdose.

\section{CONCLUSION}

In the present case, G6PD deficient child was given three different acetaminophen brands simultaneously by mother resulting in acetaminophen overdose and subsequent development of haemolysis. It revealed the lack of parental knowledge that three different brands contain the same drug. This report highlights the necessity of parental education about the medications that can trigger haemolysis in G6PD deficient children and also about the availability of different brands of the same drug in the market.

\section{ACKNOWLEDGEMENT}

The authors would like to thank the staffs and the students of Paediatrics department and Clinical Pharmacy department, JSS Hospital, Mysuru for their support and encouragement.

\section{CONFLICT OF INTEREST}

The authors declare no conflict of interest.

\section{ABBREVIATIONS}

G6PD: Glucose-6-Phosphate Dehydrogenase; WHO: World Health Organization; NSAIDs: Non-Steroidal Anti-Inflammatory Drugs; RBCs: Red Blood Cells; NAPQI: N-acetyl-P-Benzoquinone Imine; NADP+: Nicotinamide Adenine Dinucleotide Phosphate; NADPH: Nicotinamide Adenine Dinucleotide Phosphate Hydrogen; ORS: Oral Rehydration Solution; Ig: Immunoglobulin; NRBC: Nucleated Red Blood Cell; PCT: Procalcitonin; PDW: Platelet Distribution Width; RDW: Red Cell Distribution Width; MCH: Mean Cell Haemoglobin; MCHC: Mean Cell Haemoglobin Concentration; PCV: Packed Cell Volume; ESR: Erythrocyte Sedimentation Rate; GSH: Glutathione; GSSH: Oxidized Glutathione. 


\section{REFERENCES}

1. Cappellini MD, Fiorelli GE. Glucose-6-phosphate dehydrogenase deficiency Lancet. 2008;371(9606):64-74.

2. Youngster I, Arcavi L, Schechmaster R, Akayzen Y, Popliski H, Shimonov J, et al. Medications and glucose-6-phosphate dehydrogenase deficiency. Drug Saf. 2010;33(9):713-26

3. Farhud DD, Yazdanpanah L. Glucose-6-phosphate dehydrogenase (G6PD) Deficiency. Iran J Public Health. 2008;37(4):1-8.

4. Craft MD, Martin MD, Fern M. Methemoglobinemia in Acetaminophen Overdose and Glucose-6-phosphate Dehydrogenase Deficiency.

5. Mehta A, Mason PJ, Vulliamy TJ. Glucose-6-phosphate dehydrogenase deficiency. Best Pract Res Clin Haematol. 2000;13(1):21-38.

6. Wright RO, Perry HE, Woolf AD, Shannon MW. Hemolysis after acetaminophen overdose in a patient with glucose-6-phosphate dehydrogenase deficiency. $J$ Toxicol Clin Toxicol. 1996;34(6):731-4.

7. Sklar GE. Hemolysis as a Potential Complication of Acetaminophen Overdose in a Patient with Glucose-6-Phosphate Dehydrogenase Deficiency. Pharmacotherapy. 2002;22(5):656-8.

8. Ruha AM, Selden B, Curry S. Hemolytic anemia after acetaminophen overdose in a patient with glucose-6-phosphate dehydrogenase deficiency. Am J Med. $2001 ; 110(3): 240-1$

9. Cayanis E, Gomperts ED, Balinsky D, Disler P, Myers A. G6PD Hillbrow: A New Variant of Glucose-6-Phosphate Dehydrogenase Associated with Drug-induced Haemolytic Anaemia. Br J Haematol. 1975;30(3):343-50.

10. Rickner SS, Cao D, Simpson SE. Hemolytic crisis following acetaminophen overdose in a patient with G6PD deficiency. Clin Toxicol. 2017;55(1):74.

11. Beutler E. G6PD deficiency. Blood. 1994;84(11):3613-36.

Article History: Submission Date : 13-11-2018; Revised Date : 05-01-2019; Acceptance Date : 17-02-2019.

Cite this article: Panachiyil GM, Tirin B, Juny S, Dhati MR. A Case Report of Haemolysis in a G6PD Deficient Child due to Acetaminophen Overdose Resulted from Parental Unawareness. J Young Pharm. 2019;11(2):224-6. 